\title{
Decoupling of regional neural activity and inter-regional functional connectivity in Alzheimer's disease: a simultaneous PET/MR study
}

\author{
Somayeh Maleki Balajoo ${ }^{1,2,3} \cdot$ Farzaneh Rahmani $^{4} \cdot$ Reza Khosrowabadi $^{5} \cdot$ Chun Meng $^{6} \cdot$ Simon B. Eickhoff ${ }^{2,3}$. \\ Timo Grimmer ${ }^{7} \cdot$ Mojtaba Zarei $^{1,8,9} \cdot$ Alexander Drzezga $^{10,11,12} \cdot$ Christian Sorg $^{7,13,14} \cdot$ Masoud Tahmasian $^{1,2,3}$ (1)
}

Received: 17 August 2021 / Accepted: 13 January 2022 / Published online: 24 February 2022

(c) The Author(s) 2022

\begin{abstract}
Purpose Alzheimer's disease (AD) and mild cognitive impairment (MCI) are characterized by both aberrant regional neural activity and disrupted inter-regional functional connectivity (FC). However, the effect of AD/MCI on the coupling between regional neural activity (measured by regional fluorodeoxyglucose imaging (rFDG)) and inter-regional FC (measured by resting-state functional magnetic resonance imaging (rs-fMRI)) is poorly understood.

Methods We scanned 19 patients with MCI, 33 patients with AD, and 26 healthy individuals by simultaneous FDG-PET/rsfMRI and assessed rFDG and inter-regional FC metrics (i.e., clustering coefficient and degree centrality). Next, we examined the potential moderating effect of disease status (MCI or AD) on the link between rFDG and inter-regional FC metrics using hierarchical moderated multiple regression analysis. We also tested this effect by considering interaction between disease status and inter-regional FC metrics, as well as interaction between disease status and rFDG.

Results Our findings revealed that both rFDG and inter-regional FC metrics were disrupted in MCI and AD. Moreover, AD altered the relationship between rFDG and inter-regional FC metrics. In particular, we found that AD moderated the effect of inter-regional FC metrics of the caudate, parahippocampal gyrus, angular gyrus, supramarginal gyrus, frontal pole, inferior temporal gyrus, middle frontal, lateral occipital, supramarginal gyrus, precuneus, and thalamus on predicting their rFDG. On the other hand, AD moderated the effect of rFDG of the parietal operculum on predicting its inter-regional FC metric.

Conclusion Our findings demonstrated that $\mathrm{AD}$ decoupled the link between regional neural activity and functional segregation and global connectivity across particular brain regions.
\end{abstract}

Keywords Alzheimer's disease $\cdot$ Mild cognitive impairment $\cdot$ PET/MR $\cdot$ Neural activity $\cdot$ Functional connectivity $\cdot$ Graph analysis

\section{Introduction}

Alzheimer's disease (AD) and mild cognitive impairment (MCI), a syndrome at risk for $\mathrm{AD}$, are characterized by progressive cognitive dysfunctions due to neuronal loss and reduced overall neural activity, which can be indirectly quantified through fluorodeoxyglucose-positron emission

This article is part of the Topical Collection on Neurology Dementia

Christian Sorg and Masoud Tahmasian contributed equally to this work.

Masoud Tahmasian

m.tahmasian@fz-juelich.de

Extended author information available on the last page of the article tomography (FDG-PET) [1]. Regional glucose metabolism ( $\mathrm{rFDG}$ ) is associated with cortical atrophy and betaamyloid/tau protein accumulation, which are hallmarks of AD pathology [2,3]. As $80 \%$ of neural metabolic activity is dedicated to synaptic signaling [4], intrinsic functional connectivity (FC) measured by resting-state functional magnetic resonance imaging (rs-fMRI) might indicate a conjugate in-/decreased blood oxygenation level-dependent (BOLD) signaling between the brain regions. Topological features of these FC networks can be modeled through graph theory analyses, in which brain regions are considered "nodes," and FC between them as "edges" of the graph [5]. Several studies demonstrated FC alterations and topological changes, mainly in the default mode network (DMN), along the trajectory of AD [6-9]. However, the effect of AD/MCI on the coupling between regional neural activity and inter-regional 
FC metrics is not well-addressed in literature. Simultaneous assessment of rFDG and FC has a great potential to identify how disease can alter their coupling, which might shed light on the neurobiological mechanisms of AD.

Empirical evidence and computational modeling [10-12] suggested that higher local metabolism (as a proxy for local activity) in a particular brain region increases its sensitivity to afferent input from other regions and thus determines the likelihood of inter-regional FC (as a proxy for synchronous fluctuations of BOLD signal between the brain regions). A recent study found synchrony and coupling between the neural dynamics of glucose metabolism and the BOLD hemodynamic response [13], suggesting a strong relationship between $\mathrm{rFDG}$ and FC. In healthy brains, up to $18 \%$ of intersubject variabilities in whole-brain glucose metabolism can be explained by differences in FC [14]. Moreover, it has been shown that increased FC and higher functional clustering are associated with a non-linear increase in regional metabolism in healthy individuals [14]. This association, however, could be disturbed in AD, where rFDG is no longer linked with regional FC in the posterior associational cortices [15]. Here, we aimed to address whether and how AD/MCI (as a clinical diagnosis of disease status) affects the coupling between $\mathrm{rFDG}$ and inter-regional FC metrics characteristics using simultaneous acquisition of FDG-PET and rs-fMRI in healthy control (HC) subjects and patients with MCI and AD. Initial results of this study were already uploaded as a preprint in BioRxiv (https://doi.org/10.1101/642629).

\section{Methods}

\section{Participants}

Thirty-three patients with mild AD-dementia, 19 patients with MCI, and $26 \mathrm{HC}$ subjects were recruited in this crosssectional study. Patients were randomly selected from outpatient memory clinic of the Department of Psychiatry and Psychotherapy of Klinikum rechts der Isar, Technical University of Munich (TUM). Diagnosis of AD or MCI was determined using Clinical Dementia Rating (CDR) and neuropsychological testing batteries based on criteria established by Consortium to Establish a Registry for Alzheimer's disease CERAD. HC subjects were also recruited through word-of-mouth advertising in Munich. This study was approved by the TUM ethics committee in line with the institute's Human Research Committee guidelines and conformed to standards of the declaration of Helsinki. Written informed consent was obtained from all participants after providing detailed information about this study. Of note, as our $\mathrm{AD} / \mathrm{MCI}$ patients did not have amyloid-/tau-PET imaging, "AD/MCI effect" solely refers to the presence or absence of clinical diagnosis here.

\section{Data acquisition and preprocessing}

Imaging data acquisition included structural MRI (T1 weighted), rs-fMRI, and FDG-PET, which were simultaneously acquired on a Biograph hybrid PET/MR scanner (Siemens, Erlangen, Germany). Images were preprocessed and analyzed according to previously published and standardized protocols (Supplemental Methods, Sect. 1) [8, 16, 17]. After removing eight subjects due to excessive motion ( $>2 \mathrm{~mm}$ translation or $2^{\circ}$ rotation), 30 patients with mild AD-dementia, 16 patients with MCI, and $24 \mathrm{HC}$ subjects were included for further analyses (Table 1).

\section{FDG-PET data analysis}

The average glucose uptake was computed for 112 cortical and subcortical regions obtained from Harvard-Oxford atlas [18] in MNI space from each subject-specific preprocessed FDG-PET data. Afterward, we normalized the mean glucose uptake value of each region by dividing it to the average glucose uptake of the pons of the same subject, as choosing the pons (as a reference region) is sensitive to detect hypometabolic changes associated with $\operatorname{AD}[19,20]$.

\section{rs-fMRI data analysis}

The average BOLD signal of 112 regions from Harvard-Oxford atlas was extracted, and the inter-regional FCs were modeled based on graph theory analysis, where the atlas regions considered as nodes and FC between them
Table 1 Demographic and clinical data of included participants

\begin{tabular}{lllll}
\hline & HC & MCI & AD & $p$-value \\
\hline Mean age, year $(S D)$ & $54.71(9.99)$ & $70.19(6.65)$ & $70.5(8.50)$ & $<0.001$ \\
$N$ (female) & $24(9)$ & $16(12)$ & $30(11)$ & 0.03 \\
Mean MMSE score $(S D)$ & $29.44(1.10)$ & $26.64(2.20)$ & $22.68(4.40)$ & $<0.001$ \\
Mean CREAD NAB total $(S D)$ & $86(8.23)$ & $67.89(11.54)$ & $53.67(11.94)$ & $<0.001$ \\
\hline
\end{tabular}

Abbreviations: $H C$, healthy control; $M C I$, mild cognitive impairment; $A D$, Alzheimer disease; $M M S E$, Mini-Mental State Examination; CERAD, Consortium to Establish a Registry for Alzheimer's Disease neuropsychological assessment battery. Group comparisons were done based on analysis of variance, $p<0.05$ as a threshold of significance, except for sex (Kruskal-Wallis test) 
(using absolute values of Pearson's correlation coefficients) as edges. Finally, a $112 \times 112$ symmetric undirected weighted matrix representing individual whole-brain interregional FC was computed for each subject. From a topological perspective, each subject has a different topology in terms of connection density between the brain regions. The connection density in a graph is defined as a ratio of number of existing edges to all possible edges [21]. Difference in connection density, in turn, influences most of the extracted topological metrics within a graph [22]. Thus, it is necessary to implement a matching strategy between FC graphs, prior to statistical analyses between the three groups [23]. Accordingly, we thresholded adjacency matrix of each subject for density range from 0.01 to 0.40 (with intervals of 0.01), as suggested earlier [24]. It means that the adjacency matrix of each subject was thresholded 40 times with different connection densities to ensure that the topology variability across all subjects was considered in the analysis. We then characterized the organization of brain regions in terms of clustering coefficient (CC; segregated role of node) [25] and degree centrality (DC; centralized role of node) [26] (Supplemental Methods, Sect. 2). Of note, CC and DC are two commonly used inter-regional FC topological metrics, representing local segregation and centrality of individual nodes, respectively [27]. Put differently, CC quantifies how well a certain node has formed locally segregated processing modules (i.e., clusters) around itself, while DC gives a measure of the centrality of a node in terms of interacting with other nodes within and outside the regional clusters and how well it is contributing to regional segregation and global integration of the network [5]. To assess the inter-regional FC metrics, we calculated those metrics using the Brain Connectivity Toolbox [5]. Finally, the integral values of CC and DC across the all thresholded adjacency matrices for each subject were used for between groups' comparison, as recommended previously [28].

\section{Group comparison and statistical analyses}

Between groups' comparisons based on the analysis of covariance (ANCOVA) on rFDG and inter-regional FC metrics (i.e., CC/DC) were performed for all 112 regions, while age and sex of subjects were considered the covariates of no-interest. The results were corrected for multiple comparisons using $\mathrm{N}$-region statistical comparison, as described previously [29]. The significance threshold was calculated using $1 /$ (number of regions), $p$-value $<1 / 112=0.009$. Post hoc analyses were then conducted for rFDG, CC, and DC utilizing permutation test with 100,000 permutations, and significance threshold for post hoc test was calculated using $p$-value $=1 /$ (number of tests) to correct for potential falsepositive errors.

\section{Cross-modality analysis}

To evaluate the moderating effect of disease (i.e., MCI/AD) on the link between rFDG the inter-regional FC metrics, we applied the hierarchical moderated multiple regression (HMMR) analysis (Supplemental Methods, Sect. 3; SIFig. 1) and focused on the regions in which rFDG or CC/ DC were affected by diseases (Fig. 1). To assess our hypothesis about the effects of disease on the relationship between rFDG and inter-regional FC metrics, we tested this effect by considering interaction between disease status and interregional $\mathrm{FC}$ metrics and also interaction between disease status and rFDG. In the first model, both CC/DC and disease status and the interaction term as " $\mathrm{CC} / \mathrm{DC} \times$ disease status" were entered to a model to predict $\mathrm{rFDG}$ as a response variable. In the second model, rFDG and disease status and the interaction term as " $\mathrm{FDG} \times$ disease status" were entered to a model to predict $\mathrm{CC} / \mathrm{DC}$ as a response variable. We also entered age and sex as the covariate of no-interest in the HMMR analyses. To evaluate whether the interaction term in both models is meaningful, we tested whether adding the interaction term increased the variance explained by the model in successive regression steps $\left(\Delta R^{2}\right.$, according to the available guidelines [30, 31]). These results were also corrected for multiple comparisons using family wise error (FWE, $p$-value $<0.05$ ).

\section{Results}

\section{Regional glucose metabolism alterations along the trajectory of disease}

Comparing rFDG between groups (controlling for age and sex), we found significantly reduced metabolism in the DMN areas including the bilateral posterior cingulate cortex, angular gyri, left parahippocampal gyrus, left middle frontal cortex, and left precuneus cortex in AD patients compared to $\mathrm{HC}$ subjects. There were also hypometabolism in $\mathrm{AD}$ patients compared to $\mathrm{HC}$ subjects in the subcortical regions (i.e., caudate, thalamus, nucleus accumbens), as well as in the temporal, lateral occipital, and frontal regions. We also found hypometabolism in the bilateral middle and inferior temporal gyri, bilateral lateral occipital gyri, bilateral angular gyri, and caudate in MCI patients compared to HC subjects (Fig. 1(a), SI-Table 1).

\section{Disrupted local FC network topology along the trajectory of disease}

We observed widespread alterations in CC/DC metrics between the groups (Fig. 1(1), SI-Tables 2 and 3). In particular, there was a progressive decrease of $\mathrm{CC}$ along the 


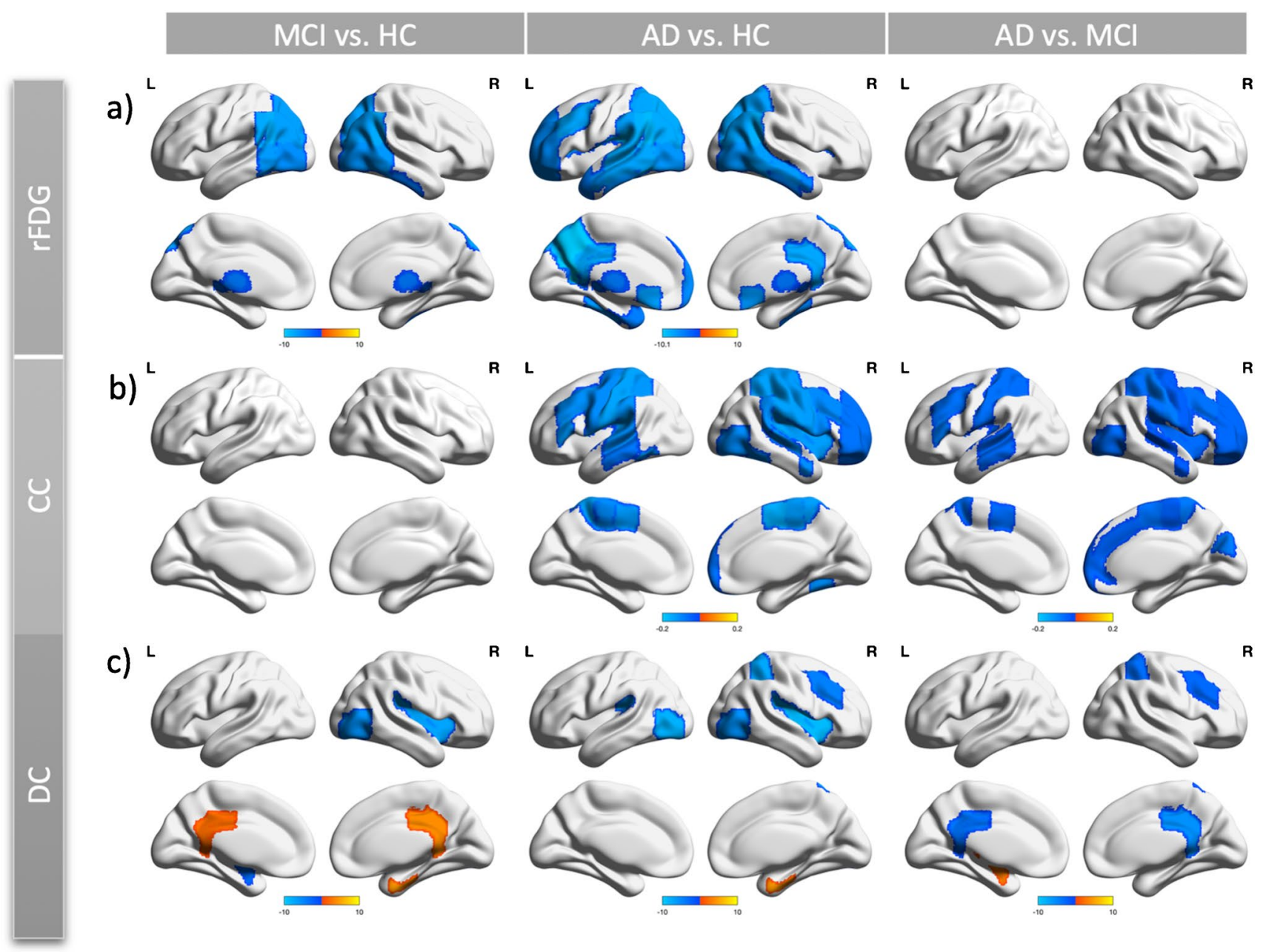

Fig. 1 Analysis of covariance on whole-brain topological measures of inter-regional FC and regional glucose metabolism for each region in Harvard-Oxford Atlas in (a) regional glucose metabolism; (b) clustering coefficient; and (c) degree centrality. Age and sex were considered as covariates of no-interest. Post hoc test: permutation test $(p<0.05,100,000$ permutations). In each group comparison, the color of significant regions was coded as the mean difference values

trajectory of disease in several regions including the bilateral middle and inferior frontal, bilateral superior, middle and inferior temporal, bilateral supramarginal, bilateral supplementary motor, bilateral occipital, bilateral Heschel, bilateral post/precentral, right temporal occipital, bilateral operculum cortex, right insular, and left angular gyri (Fig. 1(1), SI-Table 2). CC was not significantly affected in MCI patients compared to $\mathrm{HC}$ subjects. Interestingly, in MCI patients, DC was increased in the bilateral posterior cingulate cortex (PCC) and right parahippocampal gyrus and decreased in the left amygdala and brainstem compared to both $\mathrm{HC}$ subjects and patients with $\mathrm{AD}$. DC of right parahippocampal gyrus also increased in $\mathrm{AD}$ patients compared to HC subjects. Moreover, in both patients' groups, DC was decreased in the right insular, bilateral lateral occipital, bilateral parietal operculum in bilateral precentral, lateral occipital, and bilateral Heschl's gyri, and increased in of $\mathrm{rfDG} / \mathrm{CC} / \mathrm{DC}$ between each pair of groups (i.e., in $\mathrm{AD}$ vs. HC: color code $=\mathrm{CC}_{\mathrm{AD}}-\mathrm{CC}_{\mathrm{HC}}$ ). For more detailed information, see SITable 1-3. FC, functional connectivity; $A D$, Alzheimer disease; $H C$, healthy control; $M C I$, mild cognitive impairment; $C C$, clustering coefficient; $D C$, degree centrality; $r F D G$, regional glucose metabolism; $L$, left; $R$, right

the bilateral parahippocampal gyri (Fig. 1(1), SI-Table 3). The results indicated a pattern of progressive decrease in DC in the right hemisphere specifically in the superior parietal lobule, planum polare, middle frontal gyrus, parietal operculum, insular, and central opercular along the trajectory of disease (Fig. 1(1) and SI-Table 3).

\section{The moderator effect of diseases on the coupling between regional glucose metabolism and inter-regional FC metrics}

\section{Interaction between disease status and inter-regional FC metrics}

In the first model, we considered the interaction between disease status and inter-regional FC metrics to test the 


\section{Moderator effect of AD on the link between rFDG and CC}
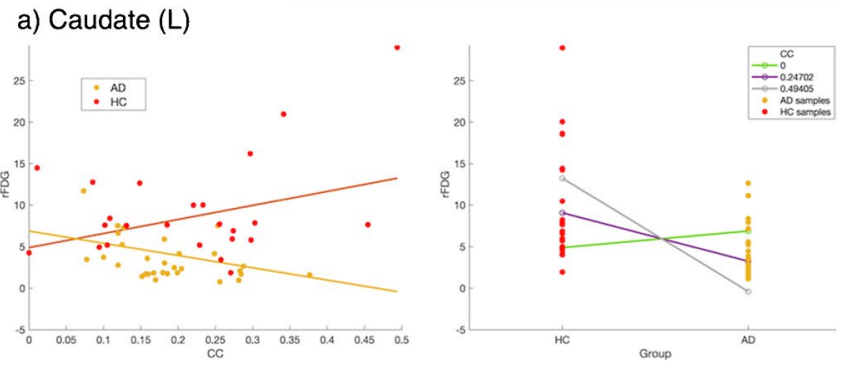

b) Parahipocampal gyrus, anterior devision (L)
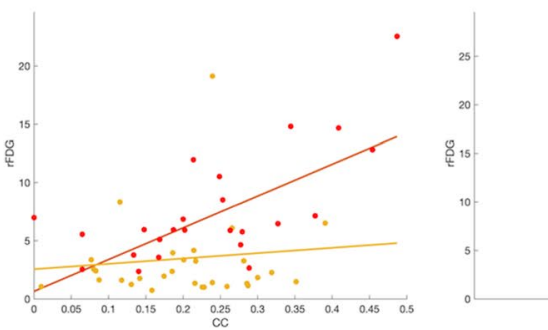

c) Angular gyrus (L)
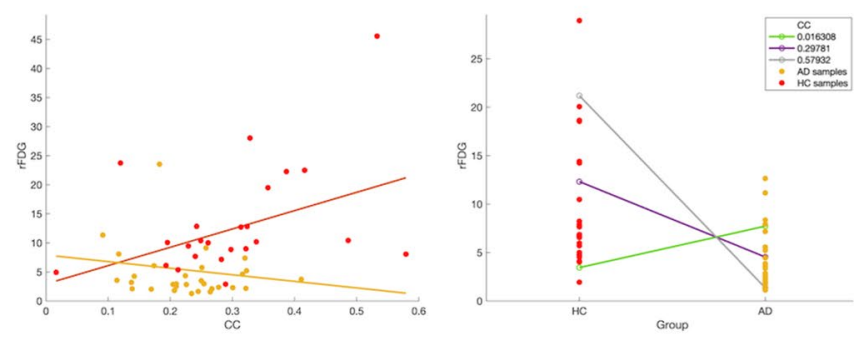

Fig. 2 Moderator effect of $\mathrm{AD}$ on the link between regional glucose metabolism and inter-regional FC metrics by considering the interaction term between disease status and inter-regional FC metrics (CC) in HMMR model. (a-f) Brain regions where significant interactions between disease status and CC predicted rFDG changes (for more information, see Table 2). In each section, figures in the first column indicate that $\mathrm{rFDG}$ increases as a function of $\mathrm{CC}$ in $\mathrm{HC}$ subjects but decreases in $\mathrm{AD}$ patients. In the second column of each section, we

potential effect of $\mathrm{AD}$ on the association between $\mathrm{rFDG}$ and $\mathrm{CC} / \mathrm{DC}$. There were significant two-way interactions between "disease status $\times \mathrm{CC}$ " only in the left hemisphere including caudate, parahippocampal gyrus, angular gyrus, supramarginal gyrus, and frontal pole and inferior temporal gyrus that predicted changes in $\mathrm{FDG}$, when comparing the $\mathrm{HC}$ and $\mathrm{AD}$ groups. Thus, $\mathrm{AD}$ moderated the effect of $\mathrm{CC}$ of the abovementioned regions on predicting their regional glucose metabolism (Fig. 2(a-f); Table 2). Only left inferior temporal region showed both significant hypometabolism and decrease in $\mathrm{CC}$ in $\mathrm{AD}$ patients compared to $\mathrm{HC}$ subjects (SI-Table 4). rFDG showed a positive association with CC in $\mathrm{HC}$ subjects, but a negative association in $\mathrm{AD}$ patients (Fig. 2(a-f); Table 2). The significant interaction between $\mathrm{CC}$ and disease status indicated that the local properties of all these regions (rFDG and CC) depend on disease status. d) Supramarginal gyrus, posterior division $(L)$

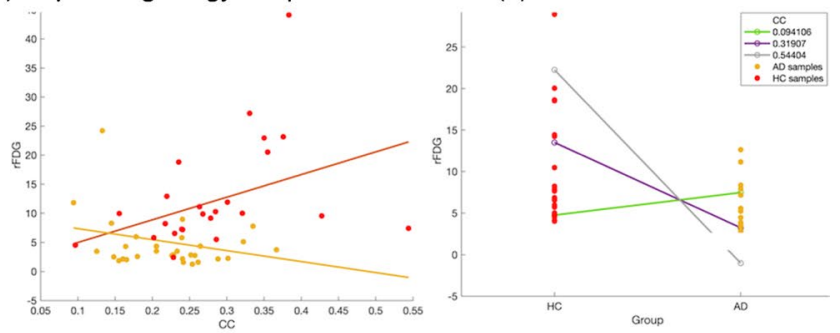

e) Frontal pole (L)

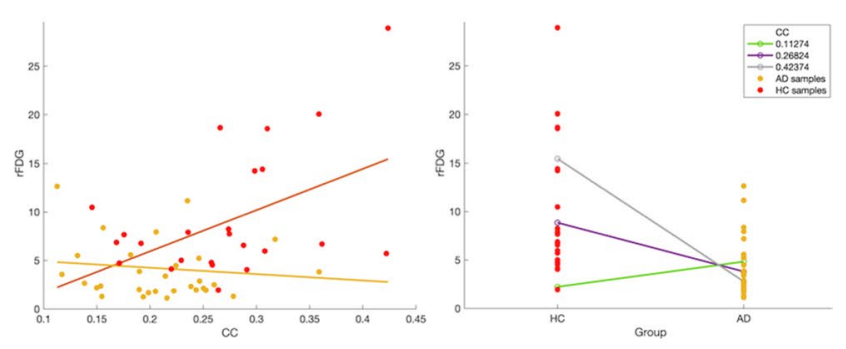

f) Inferior temporal gyrus, temporooccipital part (L)
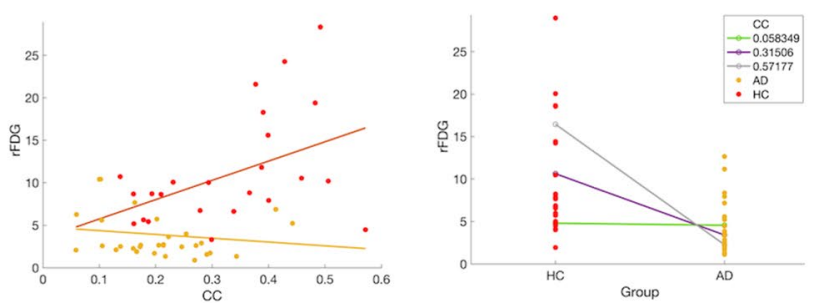

showed changes in the rFDG in both $\mathrm{HC}$ and $\mathrm{AD}$ groups, when the $\mathrm{CC}$ is fixed at three low, medium, and high levels. The lines for $\mathrm{HC}$ and $\mathrm{AD}$ groups are crossing, indicating an interaction between $\mathrm{CC}$ and disease status. $A D$, Alzheimer's disease; $H C$, healthy control; $H M M R$, hierarchical moderated multiple regression; $F C$, functional connectivity; $C C$, clustering coefficient; $r F D G$, regional glucose metabolism

No significant interaction between disease status and CC predicted rFDG alteration, when comparing the $\mathrm{AD}$ vs. $\mathrm{MCI}$ or MCI vs. HC groups.

In addition, there were also significant two-way interactions between "disease status $\times$ DC" in the bilateral thalamus, left middle frontal, left lateral occipital, left supramarginal gyrus, and the left precuneus that predicted changes in rFDG, when comparing the $\mathrm{HC}$ and $\mathrm{AD}$ groups (Fig. 3(a-f); Table 3). Our results also demonstrated significant two-way interactions between "disease status $\times D C$ " in the bilateral thalamus that predicted changes in rFDG, when comparing the $\mathrm{HC}$ and MCI groups (SI-Fig. 2; SI-Table 5). Figures 3 (a-f) and SI-Fig. 2 indicate that rFDG showed a positive association with $\mathrm{DC}$ in $\mathrm{HC}$ subjects, but a negative association in $\mathrm{AD} / \mathrm{MCI}$ patients. The significant interaction between DC and disease status indicated that the local 
Table 2 Statistical results related to the first model considering the interaction between disease status and CC to predict rFDG changes, when comparing $\mathrm{HC}$ and $\mathrm{AD}$ groups

\begin{tabular}{|c|c|c|c|c|c|c|c|}
\hline \multicolumn{2}{|c|}{ Model parameters and statistics } & \multirow{2}{*}{$\begin{array}{l}\text { Caudate (L) } \\
0.44\end{array}$} & \multirow{2}{*}{$\begin{array}{l}\text { Parahipocam- } \\
\text { pal, anterior } \\
\text { (L) } \\
0.47\end{array}$} & \multirow{2}{*}{$\begin{array}{l}\text { Angular (L) } \\
0.40\end{array}$} & \multirow{2}{*}{$\begin{array}{l}\begin{array}{l}\text { Supramarginal } \\
\text { gyrus, posterior } \\
\text { (L) }\end{array} \\
0.37\end{array}$} & \multirow{2}{*}{$\begin{array}{l}\text { Frontal pole (L) } \\
0.39\end{array}$} & \multirow{2}{*}{$\begin{array}{l}\text { Inferior temporal, } \\
\text { temporooccipital } \\
(\mathrm{L})\end{array}$} \\
\hline HMMR model ${ }^{\mathrm{a}, \mathrm{b}}$ & $R^{2}$ & & & & & & \\
\hline & Adjusted $R^{2}$ & 0.38 & 0.42 & 0.34 & 0.31 & 0.33 & 0.46 \\
\hline & $F(5,53)$ & 7.50 & 8.60 & 6.50 & 5.66 & 6.16 & 10.01 \\
\hline & $\begin{array}{l}P \text {-val- } \\
\text { ue }_{\text {model (FWE-corrected) }}\end{array}$ & $<0.0001$ & $<0.0001$ & $<0.0001$ & $<0.0001$ & $<0.0001$ & $<0.0001$ \\
\hline & $\Delta R^{2}: R^{2}$ change & 0.68 & 0.56 & 0.06 & 0.07 & 0.07 & 0.05 \\
\hline & $p$-value $_{\text {interaction term }}$ & 0.02 & 0.03 & 0.04 & 0.02 & 0.03 & 0.03 \\
\hline \multirow{5}{*}{$\begin{array}{r}\text { Standardized } \\
\text { coefficients }^{\mathrm{a}}\end{array}$} & Disease status & 0.19 & 0.20 & 0.30 & 0.51 & 0.74 & 0.11 \\
\hline & Age & -0.21 & -0.14 & -0.16 & -0.10 & -0.10 & -0.073 \\
\hline & Sex & -0.02 & 0.11 & 0.016 & 0.04 & 0.10 & 0.014 \\
\hline & $\mathrm{CC}$ & 0.90 & 1.13 & 0.94 & 1.02 & 1.18 & 1.023 \\
\hline & $\begin{array}{l}\text { Interaction (disease } \\
\text { status } \times \text { CC) }\end{array}$ & -0.93 & -0.86 & -0.81 & -1.01 & -1.08 & -0.75 \\
\hline
\end{tabular}

${ }^{a}$ Response variable: $r F D G$

${ }^{b}$ Predictors: $\mathrm{CC}$, sex, age, disease status, interaction (disease status $\times \mathrm{CC}$ )

Abbreviations: $A D$, Alzheimer's disease; $H C$, healthy control; $H M M R$, hierarchical moderated multiple regression; $C C$, clustering coefficient; $r F D G$, regional glucose metabolism

properties of all these regions ( $\mathrm{rFDG}$ and DC) depend on disease status. No significant interaction between disease status and DC could predict rFDG alteration, when comparing the $\mathrm{AD}$ vs. MCI groups.

\section{Interaction between disease status and rFDG}

In the second model, we considered the interaction between disease status and rFDG to test the potential effect of $\mathrm{AD}$ on the association between rFDG and CC/DC. Results showed that only in the left parietal operculum a significant interaction between disease status and rFDG predicted DC alteration, when comparing the $\mathrm{AD}$ vs. HC groups (Fig. 4; Table 4). The left parietal operculum showed significant hypometabolism and decrease in DC in AD patients compared to HC subjects (SI-Table 4). In Fig. 4, DC showed a negative association with $\mathrm{rFDG}$ in $\mathrm{HC}$ subjects, but a positive association in $\mathrm{AD}$ patients. The significant interaction between rFDG and disease status indicated that the local properties of this region ( $\mathrm{rFDG}$ and $\mathrm{DC}$ ) depend on disease status.

\section{Discussion}

We examined the moderator effect of disease (i.e., MCI/ $\mathrm{AD}$ ) on the coupling between regional neural activity and topological measures of inter-regional FC. Our findings demonstrated the following: (i) cortical hypometabolism along with reduced CC/DC in several brain regions including the main hubs of DMN in MCI and AD patients, although in both patients groups, DC was increased in the right parahippocampal gyrus (Fig. 1); (ii) significant two-way interactions between inter-regional FC metrics and "disease status" predicted rFDG alterations in various brain regions in the left hemisphere including parahippocampal gyrus, angular gyrus, caudate, supramarginal gyrus, frontal pole, inferior temporal gyrus, middle frontal, lateral occipital, supramarginal gyrus, precuneus, and the bilateral thalamus, when comparing the $\mathrm{HC}$ and $\mathrm{AD}$ groups (Figs. 2, 3); (iii) a significant two-way interaction between DC and disease status predicted rFDG alterations in the bilateral thalamus, when comparing the HC and MCI groups (SI-Fig. 2); (iv) a significant two-way interaction between rFDG and disease status predicted DC alterations only in the left parietal operculum, when comparing the $\mathrm{HC}$ and $\mathrm{AD}$ groups (Fig. 4). These findings suggest that AD disrupts a coupling between $\mathrm{rFDG}$ and topological measures of inter-regional FC in particular regions including the main hubs of DMN.

\section{Hypometabolism and disrupted inter-regional FC topology in MCI/AD}

Hypometabolism and FC disruption, mainly in the posterior part of DMN, are well-documented features of $\mathrm{AD}$ that are suggested to be strongly linked with grey matter 


\section{Moderator effect of AD on the link between rFDG and DC}

a) Thalamus (L)

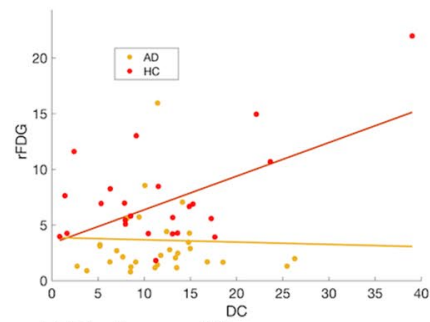

b) Thalamus (R)

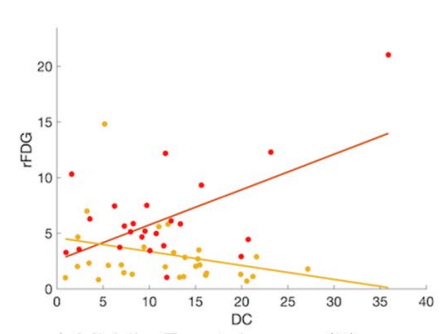

c) Middle Frontal gyrus ( $L$ )

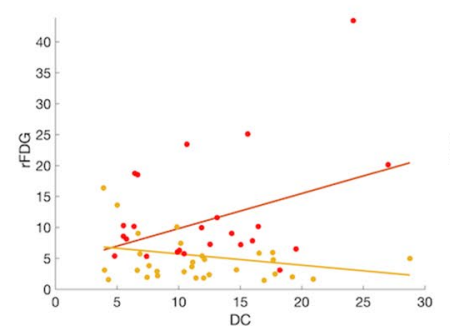

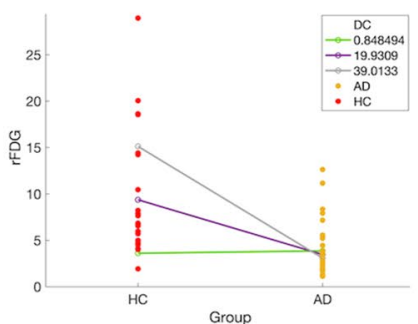

Group

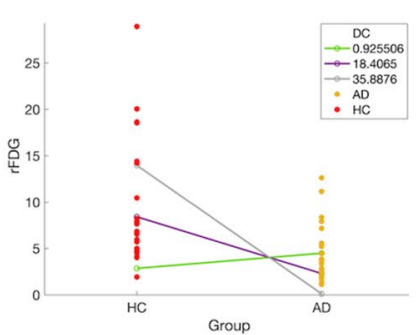

Group

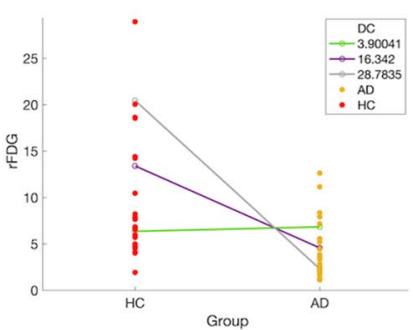

Group

d) Lateral Occipital cortex, superior division (L)

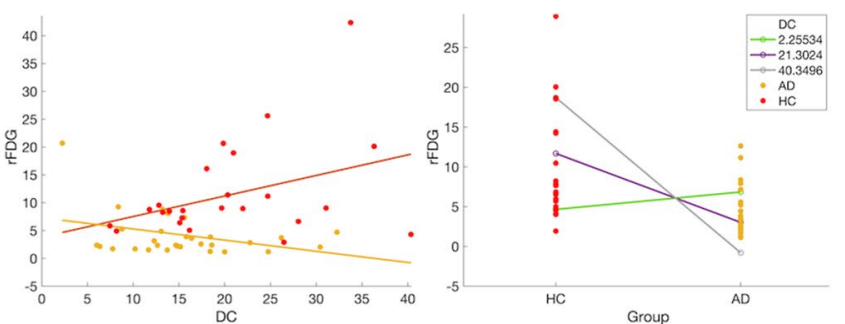

e) Supramarginal gyrus, posterior division (L)
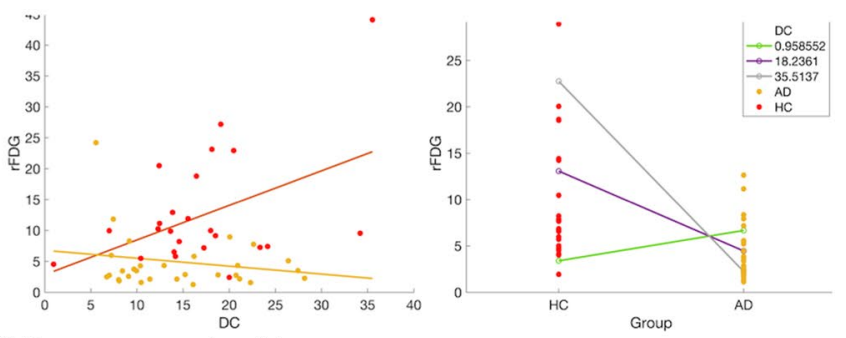

f) Precuneous cortex (L)
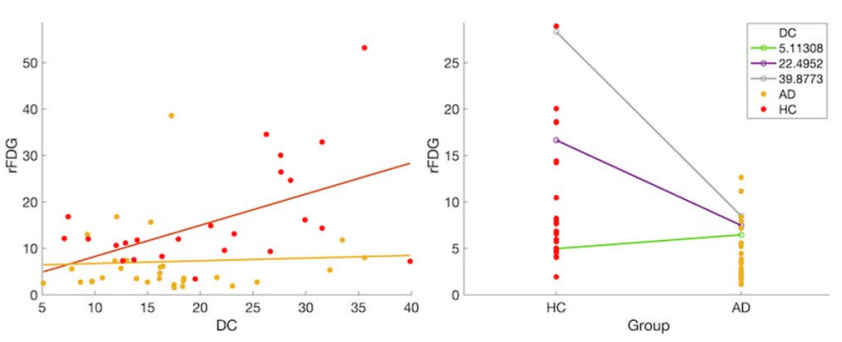

In second column, we presented that changes in the DC in both $\mathrm{HC}$ and $\mathrm{AD}$ groups, when the $\mathrm{rFDG}$ is fixed at three low, medium, and high levels. The lines for $\mathrm{HC}$ and $\mathrm{AD}$ groups are crossing, indicating an interaction between rFDG and disease status. $A D$, Alzheimer's disease; $H C$, healthy control; $H M M R$, hierarchical moderated multiple regression; $F C$, functional connectivity; $D C$, degree centrality; $r F D G$, regional glucose metabolism

precipitate the age-associated tau deposition in this region, leading to a loss of white matter integrity and structural dysconnectivity between the temporal lobe and posteromedial cortex [34]. It has been hypothesized that amyloid beta accumulation in the posteromedial cortex disrupts its long-range FC with the medical temporal lobe, which in turn gives rise to disinhibition-like pattern and increased local metabolism in the medical temporal lobe including the hippocampal formation and parahippocampal gyrus. These alterations enhance regional amyloid and tau deposition in the medial temporal lobe, further FC disruption to the remote areas, and finally lead widespread atrophy $[7,8,35]$. Interestingly, normalizing hippocampal hyperactivity, measured by task fMRI, improves task-related memory performance and has a therapeutic potential in MCI patients [36]. 
Table 3 Statistical results related to the first model considering the interaction between disease status and DC to predict rFDG changes, when comparing $\mathrm{HC}$ and $\mathrm{AD}$ groups

\begin{tabular}{|c|c|c|c|c|c|c|c|}
\hline \multicolumn{2}{|c|}{ Model parameters and statistics } & \multirow{2}{*}{$\begin{array}{l}\text { Thalamus (L) } \\
0.43\end{array}$} & \multirow{2}{*}{$\begin{array}{l}\text { Thalamus (R) } \\
0.44\end{array}$} & \multirow{2}{*}{$\begin{array}{l}\begin{array}{l}\text { Middle } \\
\text { frontal } \\
\text { gyrus (L) }\end{array} \\
0.37\end{array}$} & \multirow{2}{*}{$\begin{array}{l}\text { Lateral occipital } \\
\text { cortex, superior } \\
\text { (L) }\end{array}$} & \multirow{2}{*}{$\begin{array}{l}\text { Supramarginal } \\
\text { gyrus, posterior } \\
\text { (L) }\end{array}$} & \multirow{2}{*}{$\begin{array}{l}\text { Precune- } \\
\text { ous cortex } \\
\text { (L) }\end{array}$} \\
\hline HMMR model ${ }^{a, b}$ & $R^{2}$ & & & & & & \\
\hline & Adjusted $R^{2}$ & 0.37 & 0.38 & 0.31 & 0.30 & 0.33 & 0.31 \\
\hline & $F(5,53)$ & 7.31 & 7.39 & 5.69 & 5.61 & 6.19 & 5.78 \\
\hline & $\begin{array}{l}P \text {-val- } \\
\text { ue }_{\text {model (FWE-corrected) }}\end{array}$ & $<0.0001$ & $<0.0001$ & $<0.0001$ & $<0.0001$ & $<0.0001$ & $<0.0001$ \\
\hline & $\Delta R^{2}: R^{2}$ change & 0.06 & 0.15 & 0.08 & 0.08 & 0.08 & 0.05 \\
\hline & $p$-value interaction term & 0.03 & 0.001 & 0.02 & 0.02 & 0.01 & 0.05 \\
\hline \multirow{5}{*}{$\begin{array}{l}\text { Standardized coef- } \\
\text { ficients }^{\mathrm{a}}\end{array}$} & Disease status & 0.06 & 0.26 & 0.23 & 0.23 & 0.24 & 0.22 \\
\hline & Age & -0.17 & -0.12 & -0.17 & -0.04 & -0.07 & -0.09 \\
\hline & Sex & 0.06 & 0.11 & 0.004 & 0.003 & 0.006 & 0.14 \\
\hline & $\mathrm{DC}$ & 1.01 & 1.39 & 1.03 & 1.02 & 1.11 & 1.03 \\
\hline & $\begin{array}{l}\text { Interaction (disease } \\
\text { status } \times \text { DC) }\end{array}$ & -0.87 & -1.45 & -1.10 & -0.97 & -1.10 & -0.80 \\
\hline
\end{tabular}

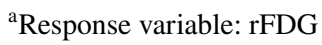

${ }^{\mathrm{b}}$ Predictors: DC, sex, age, disease status, interaction (disease status $\times \mathrm{DC}$ )

Abbreviations: $A D$, Alzheimer's disease; $H C$, healthy control; $H M M R$, hierarchical moderated multiple regression; $D C$, degree centrality; $r F D G$, regional glucose metabolism

\section{Moderator effect of AD on the link between rFDG and DC}

\section{Parietal Operculum cortex (L)}

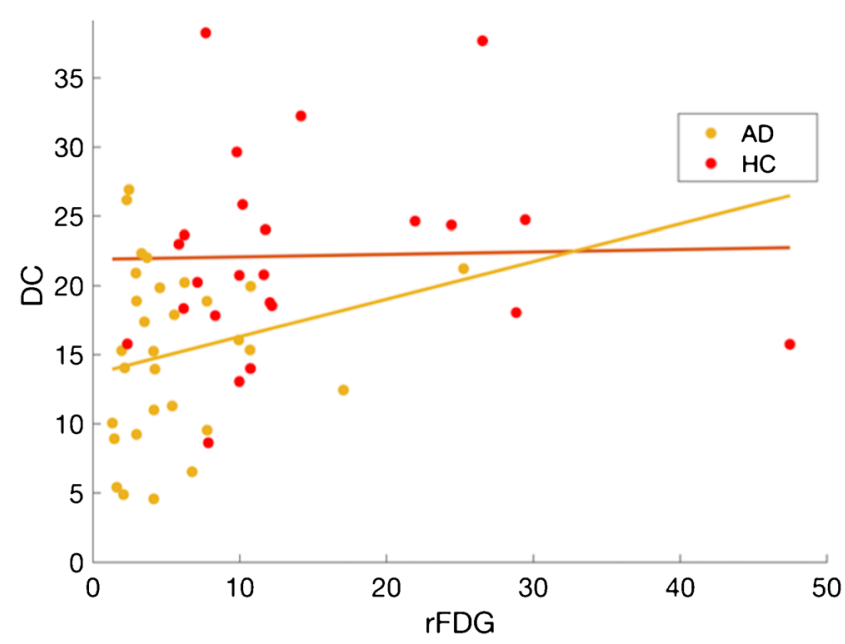

Fig. 4 Moderator effect of $\mathrm{AD}$ on the link betweenregional glucose metabolism and inter-regionalFC metrics by considering the interaction term between disease status and rFDGin HMMR model. Results showed thatonly in the left parietal operculum, a significantinteraction between disease status and rFDG predicted DC alteration, when comparing ADvs. HC groups (for more information, see Table 4). First column shows that DC decreases as a function of rFDG in HC subjects but increases in ADpatients in the left parietaloperculum.

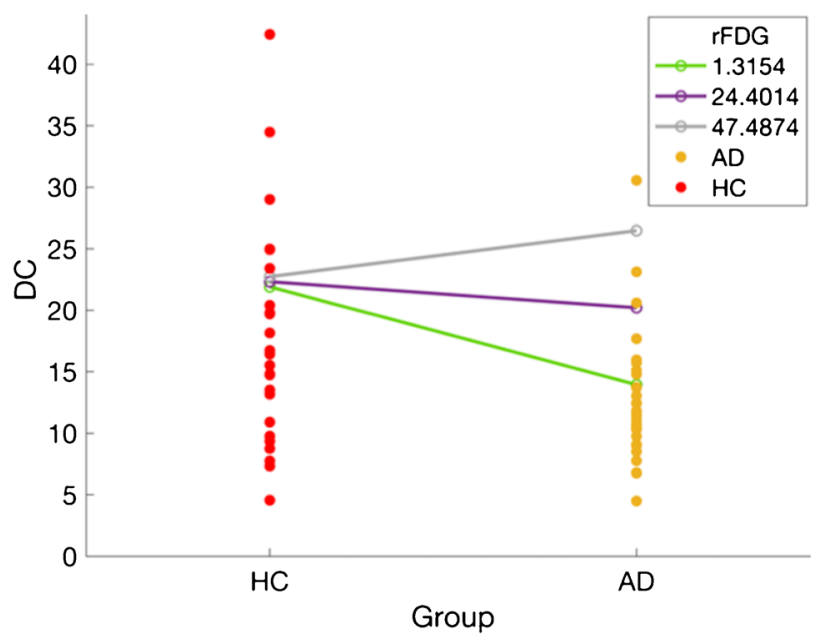

In second column, wepresented that changes in the DC in both HC and $\mathrm{AD}$ groups, when the rFDG isfixed at three low, medium and high levels. The lines for $\mathrm{HC}$ and $\mathrm{AD}$ groups arecrossing, indicating an interaction between rFDG and disease status. $A D$, Alzheimer's disease; $H C$, healthy control; $H M M R$, hierarchical moderated multipleregression; $F C$, functionalconnectivity; $D C$, degree centrality; $r F D G$, regional glucose metabolism 
Table 4 Statistical results related to the second model considering the interaction between disease status and rFDG to predict DC changes, when comparing $\mathrm{HC}$ and $\mathrm{AD}$ groups

\begin{tabular}{lll}
\hline Model parameters and statistics & & $\begin{array}{l}\text { Parietal oper- } \\
\text { culum cortex } \\
(\mathrm{L})\end{array}$ \\
\hline HMMR model $^{\mathbf{a}, \mathbf{b}}$ & $R^{2}$ & 0.93 \\
& Adjusted $R^{2}$ & 0.92 \\
& $F(5,53)$ & 130.89 \\
& $P$-value model (FWE-corrected) & $<0.0001$ \\
& $\Delta R^{2}: R^{2}$ change & 0.69 \\
Standardized coefficients $^{\text {a }}$ & $p$-value & $<0.0001$ \\
& Disteraction term & -0.74 \\
& Age & -0.11 \\
& Sex & -0.07 \\
& rFDG & -0.20 \\
& Interaction (disease status $\times$ rFDG) & 0.91 \\
\hline
\end{tabular}

${ }^{\mathrm{a}}$ Response variable: DC

bPredictors: (constant), rFDG, sex, age, disease status, interaction (disease status $\times$ rFDG)

Abbreviations: $A D$, Alzheimer's disease; $H C$, healthy control; $H M M R$, hierarchical moderated multiple regression; $D C$, degree centrality; $r F D G$, regional glucose metabolism

\section{AD decoupled regional neural activity and inter-regional FC topology}

We found that AD (as a clinical diagnosis), but not MCI, altered the physiological coupling between CC and rFDG in the left caudate, parahippocampal gyrus, angular gyrus, supramarginal gyrus, frontal pole, and inferior temporal gyrus (Fig. 2(a-f); Table 2). Moreover, both MCI and AD altered the coupling between DC and rFDG in the bilateral thalamus, left middle frontal, left lateral occipital, left supramarginal gyrus, and the left precuneus (Fig. 3(a-f) and SI-Fig. 2; Table 3 and SI-Table 5). Using an alternative model, we observed that interaction between $\mathrm{AD}$ and $\mathrm{rFDG}$ predicted DC alteration only in the left parietal operculum (Fig. 4; Table 4). These findings collectively indicated that $\mathrm{AD}$ interacts with the link between $\mathrm{CC} / \mathrm{DC}$ and rFDG. The altered association between rFDG and inter-regional FC in AD has been reported previously. For example, Marchitelli and colleagues assessed the link between FDG-PET and different rs-fMRI measures (i.e., fractional amplitude of low-frequency fluctuations (fALFF), regional homogeneity, and group independent component analysis (ICA)) on patients with MCI/ $\mathrm{AD}$ and also $\mathrm{HC}$ subjects using Spearman partial correlations. The correlation between FDG and ICA maps revealed the highest overlap in the posterior part of DMN, while FDG-fALFF correlation pattern showed the lowest overlap, mainly in the frontal and non-DMN regions including the thalamus and caudate nucleus, suggesting rFDG-FC decoupling within and outside DMN areas in $\mathrm{MCI} / \mathrm{AD}$ patients [15]. Another study demonstrated that local amyloid beta pathology might be responsible for such decoupling between rFDG and inter-regional FC within the DMN in AD [37]. In particular, Scherr and colleagues found that $\mathrm{FFDG}$ progressively decoupled from FC in the posterior DMN and degree of this decoupling associated with amyloid beta load. The authors identified "rFDG-FC coupling" as the only significant variable, which predicted cognitive status of the patients with early and late MCI and AD [37]. Similarly, our results support their findings by showing the adverse effect of $A D$ on the normal coupling between rFDG and CC in the DMN. We found widespread and congruent alterations in $\mathrm{rFDG}$ and interregional FC metrics in the main hubs of DMN including the parahippocampal gyrus, precuneus, angular gyrus, and the inferior temporal gyri, suggesting that AD pathology in the DMN can be (at least partly) accountable for the changes observed in our current study. A longitudinal study demonstrated that severity of amyloid deposition in the regions with high degree of amyloid beta pathology (i.e., in the posterior DMN) can predict progressive hypometabolism in remote but functionally connected areas, with minimal amyloid pathology [38]. It is worthy to mention that amyloid beta and tau pathology interact in their regulation of synaptic function. Synaptic tau and amyloid deposition mutually precipitate signaling pathways that culminate in progressive synaptic dysfunction and loss $[39,40]$. Indeed, it has been demonstrated that in individuals with normal amyloid beta level, FC has an inverse correlation with degree of tau deposition [41]. A similar model demonstrated that cascading network failure is mediated by amyloid deposition in the DMN along 
with global tau deposition [42]. Based on these findings and a recently proposed model by our group [7], it seems that amyloid beta and tau pathology might be important driving forces for alterations in $\mathrm{rFDG}$, inter-regional FC metrics, and rFDG-FC decoupling in $\mathrm{AD}$, which should be directly more elaborated in the future.

In addition, we identified that $\mathrm{AD}$ altered the coupling between DC and rFDG in non-DMN areas. Importantly, the important roles of caudate, thalamus, and parieto-occipital cortex have been previously highlighted in AD [43-46]. We assume that tau pathology might be responsible for rFDG and topology alterations beyond the DMN including the thalamus, supramarginal gyrus, and parietal and occipital areas. This is perhaps supported by the fact that tau deposition might be more strongly associated with rFDG decline than amyloid deposition [47]. Moreover, connectivity analysis based on tau imaging showed moderate spatial overlap, not only within the DMN, but also within the visual and language networks [48].

\section{Strengths and limitations}

A major strength of the current study was using hybrid PET/ MR scanner, which enabled us to measure both FDG-PET and rs-fMRI at the same time and provided a great opportunity to test the moderator effect of disease on the association between rFDG and inter-regional FC metrics. An important drawback of the current study, however, was the absence of amyloid and tau imaging, which could help to assess the credibility of our hypothesis regarding the role of AD pathology on rFDG and topological measures of inter-regional FC coupling. Thereby, we find it imperative for the next studies to test the pathogenic role of amyloid/ tau on metabolism-topology decoupling through simultaneous in vivo amyloid and tau PET imaging. Other limitations were the small samples and age gap between patients and HC subjects. Although we corrected the effect of age by adding it as covariate of no-interest in our regression models, the residual effect of age may still exist or has not-linear effects on functional brain networks [49]. Last but not least, our design was cross sectional, which could impede the drawing of a causal relationship between rFDG and topology changes in $\mathrm{AD}$.

\section{Conclusion}

The present study using simultaneous evaluation of FDGPET and rs-fMRI provided evidence regarding the role of $\mathrm{AD}$ on decoupling between regional neural activity and inter-regional FC alterations. In particular, we demonstrated not only regional metabolism and inter-regional FC were disrupted in patients with $\mathrm{MCI}$ and $\mathrm{AD}$, but also there is an adverse effect of $\mathrm{AD}$ on coupling between them, within, and outside the DMN. The observed abnormal neuroenergetic coupling of glucose metabolism and inter-regional FC across various brain regions/networks along the trajectory of AD provided novel insights into the pathophysiology of AD. Future longitudinal studies with larger sample size should further test the role of pathological biomarkers of $\mathrm{AD}$ (e.g., amyloid and tau proteins) on coupling between regional metabolism and functional dysconnectivity along the trajectory of disease.

Supplementary Information The online version contains supplementary material available at https://doi.org/10.1007/s00259-022-05692-1.

Acknowledgements We would like to thank all subjects for their participation in the study. Furthermore, the authors thank the staff of the Departments of Psychiatry and Psychotherapy and also Nuclear Medicine of Klinikum rechts der Isar for their help in recruitment and data collection.

Funding Open Access funding enabled and organized by Projekt DEAL.

Availability of data and material The datasets generated during and/ or analyzed during the current study are not publicly available due to ethical considerations, but will be available from the corresponding author on reasonable request.

Code availability Code can be shared upon reasonable request from the corresponding author.

\section{Declarations}

Ethics approval This study was approved by the TUM ethics committee in line with the institute's Human Research Committee guidelines and conformed to standards of the declaration of Helsinki.

Consent to participate Written informed consent was obtained from all participants after providing detailed information about this study.

Conflict of interest A. Drzezga declares the following conflict of interest: research support from Siemens Healthineers, Life Molecular Imaging, GE Healthcare, AVID Radiopharmaceuticals, and SOFIE; speaker honorary/advisory boards: Siemens Healthineers, Sanofi, GE Healthcare, Biogen, Novo Nordisk, Invicro; stock: Siemens Healthineers, Lantheus Holding, Biogen; patents: patent pending for 18F-PSMA7 (PSMA PET imaging tracer). Outside the submitted manuscript, T. Grimmer received consulting fees from Abbvie, Anavex, Biogen, Bracket, Eli Lilly, Functional Neuromodulation, Grifols, IQVIA/Quintiles, Novartis, Novo Nordisk, Noselab, NuiCare, Roche Pharma, Toyama, and Vivoryon; lecture fees from Actelion, B. Braun, Biogen, Eli Lilly, Life Molecular Imaging, Novartis, Parexel, and Roche Pharma; and grants to his institution from Actelion and Novartis. The other authors declare no competing interests.

Open Access This article is licensed under a Creative Commons Attribution 4.0 International License, which permits use, sharing, adaptation, distribution and reproduction in any medium or format, as long as you give appropriate credit to the original author(s) and the source, provide a link to the Creative Commons licence, and indicate if changes were made. The images or other third party material in this article are 
included in the article's Creative Commons licence, unless indicated otherwise in a credit line to the material. If material is not included in the article's Creative Commons licence and your intended use is not permitted by statutory regulation or exceeds the permitted use, you will need to obtain permission directly from the copyright holder. To view a copy of this licence, visit http://creativecommons.org/licenses/by/4.0/.

\section{References}

1. Minoshima S, Frey KA, Koeppe RA, Foster NL, Kuhl DE. A diagnostic approach in Alzheimer's disease using three-dimensional stereotactic surface projections of fluorine-18-FDG PET. J Nucl Med. 1995;36:1238-48.

2. Chetelat G, Desgranges B, Landeau B, Mezenge F, Poline JB, de la Sayette V, et al. Direct voxel-based comparison between grey matter hypometabolism and atrophy in Alzheimer's disease. Brain. 2008;131:60-71. https://doi.org/10.1093/brain/awm288.

3. Drzezga A, Grimmer T, Henriksen G, Stangier I, Perneczky R, Diehl-Schmid J, et al. Imaging of amyloid plaques and cerebral glucose metabolism in semantic dementia and Alzheimer's disease. Neuroimage. 2008;39:619-33. https://doi.org/10.1016/j. neuroimage.2007.09.020.

4. Attwell D, Laughlin SB. An energy budget for signaling in the grey matter of the brain. J Cereb Blood Flow Metab. 2001;21:1133-45. https://doi.org/10.1097/00004647-20011 0000-00001.

5. Rubinov M, Sporns O. Complex network measures of brain connectivity: uses and interpretations. Neuroimage. 2010;52:105969. https://doi.org/10.1016/j.neuroimage.2009.10.003.

6. Sorg C, Riedl V, Muhlau M, Calhoun VD, Eichele T, Laer L, et al. Selective changes of resting-state networks in individuals at risk for Alzheimer's disease. Proc Natl Acad Sci U S A. 2007;104:18760-5. https://doi.org/10.1073/pnas.0708803104.

7. Pasquini L, Rahmani F, Maleki-Balajoo S, La Joie R, Zarei M, Sorg C, et al. Medial temporal lobe disconnection and hyperexcitability across Alzheimer's disease stages. J Alzheimers Dis Rep. 2019;3:103-12. https://doi.org/10.3233/ADR-190121.

8. Tahmasian M, Pasquini L, Scherr M, Meng C, Forster S, MulejBratec $S$, et al. The lower hippocampus global connectivity, the higher its local metabolism in Alzheimer disease. Neurol. 2015;84:1956-63. https://doi.org/10.1212/WNL.0000000000 001575 .

9. Sanz-Arigita EJ, Schoonheim MM, Damoiseaux JS, Rombouts SA, Maris E, Barkhof F, et al. Loss of 'small-world'networks in Alzheimer's disease: graph analysis of FMRI resting-state functional connectivity. PloS one. 2010;5:e13788.

10. Hipp JF, Hawellek DJ, Corbetta M, Siegel M, Engel AK. Largescale cortical correlation structure of spontaneous oscillatory activity. Nat Neurosci. 2012;15:884-90. https://doi.org/10.1038/ nn.3101.

11. Riedl V, Bienkowska K, Strobel C, Tahmasian M, Grimmer T, Forster S, et al. Local activity determines functional connectivity in the resting human brain: a simultaneous FDG-PET/fMRI study. J Neurosci. 2014;34:6260-6. https://doi.org/10.1523/JNEUR OSCI.0492-14.2014.

12. Savio A, Fünger S, Tahmasian M, Rachakonda S, Manoliu A, Sorg $\mathrm{C}$, et al. Resting-state networks as simultaneously measured with functional MRI and PET. J Nucl Med. 2017;58:1314-7. https:// doi.org/10.2967/jnumed.116.185835.

13. Jamadar SD, Ward PGD, Close TG, Fornito A, Premaratne M, O'Brien K, et al. Simultaneous BOLD-fMRI and constant infusion FDG-PET data of the resting human brain. Sci Data. 2020;7:363. https://doi.org/10.1038/s41597-020-00699-5.
14. Tomasi D, Wang G-J, Volkow ND. Energetic cost of brain functional connectivity. Proc Natl Acad Sci. 2013;110:13642. https:// doi.org/10.1073/pnas.1303346110.

15. Marchitelli R, Aiello M, Cachia A, Quarantelli M, Cavaliere C, Postiglione A, et al. Simultaneous resting-state FDG-PET/fMRI in Alzheimer disease: relationship between glucose metabolism and intrinsic activity. Neuroimage. 2018;176:246-58. https://doi. org/10.1016/j.neuroimage.2018.04.048.

16. Scherr M, Utz L, Tahmasian M, Pasquini L, Grothe MJ, Rauschecker JP, et al. Effective connectivity in the default mode network is distinctively disrupted in Alzheimer's disease-a simultaneous resting-state FDG-PET/fMRI study. Hum Brain Mapp. 2019. https://doi.org/10.1002/hbm.24517.

17. Tahmasian M, Shao J, Meng C, Grimmer T, Diehl-Schmid J, Yousefi BH, et al. Based on the network degeneration hypothesis: separating individual patients with different neurodegenerative syndromes in a preliminary hybrid PET/MR study. J Nucl Med. 2016;57:410-5. https://doi.org/10.2967/jnumed.115.165464.

18. Keuken MC, Bazin PL, Crown L, Hootsmans J, Laufer A, MullerAxt C, et al. Quantifying inter-individual anatomical variability in the subcortex using 7 T structural MRI. Neuroimage. 2014;94:40 6. https://doi.org/10.1016/j.neuroimage.2014.03.032.

19. Verger A, Doyen M, Campion JY, Guedj E. The pons as reference region for intensity normalization in semi-quantitative analysis of brain (18)FDG PET: application to metabolic changes related to ageing in conventional and digital control databases. EJNMMI Res. 2021;11:31. https://doi.org/10.1186/s13550-021-00771-0.

20. Nugent S, Croteau E, Potvin O, Castellano CA, Dieumegarde L, Cunnane SC, et al. Selection of the optimal intensity normalization region for FDG-PET studies of normal aging and Alzheimer's disease. Sci Rep. 2020;10:9261. https://doi.org/10.1038/ s41598-020-65957-3.

21. Bullmore E, Sporns O. The economy of brain network organization. Nat Rev Neurosci. 2012;13:336-49. https://doi.org/10.1038/ nrn3214

22. Kaiser M. A tutorial in connectome analysis: topological and spatial features of brain networks. Neuroimage. 2011;57:892-907. https://doi.org/10.1016/j.neuroimage.2011.05.025.

23. van Wijk BCM, Stam CJ, Daffertshofer A. Comparing brain networks of different size and connectivity density using graph theory. PLoS ONE. 2010;5:e13701.

24. Sadeghi M, Khosrowabadi R, Bakouie F, Mahdavi H, Eslahchi C, Pouretemad H. Screening of autism based on task-free fMRI using graph theoretical approach. Psychiatry Res Neuroimaging. 2017;263:48-56. https://doi.org/10.1016/j.pscychresns.2017.02. 004.

25. van den Heuvel MP, Hulshoff Pol HE. Exploring the brain network: a review on resting-state fMRI functional connectivity. Eur Neuropsychopharmacol. 2010;20:519-34. https://doi.org/ 10.1016/j.euroneuro.2010.03.008.

26. Sporns O. Network attributes for segregation and integration in the human brain. Curr Opin Neurobiol. 2013;23:162-71. https:// doi.org/10.1016/j.conb.2012.11.015.

27. Masuda N, Sakaki M, Ezaki T, Watanabe T. Clustering coefficients for correlation networks. Frontiers in Neuroinformatics. 2018;12. https://doi.org/10.3389/fninf.2018.00007.

28. Gong G, Rosa-Neto P, Carbonell F, Chen ZJ, He Y, Evans AC. Age- and gender-related differences in the cortical anatomical network. The Journal of neuroscience : the official journal of the Society for Neuroscience. 2009;29:15684-93. https://doi.org/10. 1523/JNEUROSCI.2308-09.2009.

29. Meng C, Brandl F, Tahmasian M, Shao J, Manoliu A, Scherr M, et al. Aberrant topology of striatum's connectivity is associated with the number of episodes in depression. Brain. 2014;137:598609. https://doi.org/10.1093/brain/awt290. 
30. Aiken LS, West SG. Multiple regression: testing and interpreting interactions. Thousand Oaks, CA, US: Sage Publications, Inc.; 1991.

31. Whisman MA, McClelland GH. Designing, testing, and interpreting interactions and moderator effects in family research. J Fam Psychol. 2005;19:111-20. https://doi.org/10.1037/0893-3200. 19.1.111.

32. Pereira JB, Mijalkov M, Kakaei E, Mecocci P, Vellas B, Tsolaki $\mathrm{M}$, et al. Disrupted network topology in patients with stable and progressive mild cognitive impairment and Alzheimer's disease. Cerebral cortex (New York, NY : 1991). 2016;26:3476-93.

33. Dickerson BC, Sperling RA. Functional abnormalities of the medial temporal lobe memory system in mild cognitive impairment and Alzheimer's disease: insights from functional MRI studies. Neuropsychologia. 2008;46:1624-35. https://doi.org/10. 1016/j.neuropsychologia.2007.11.030.

34. Strain JF, Smith RX, Beaumont H, Roe CM, Gordon BA, Mishra $\mathrm{S}$, et al. Loss of white matter integrity reflects tau accumulation in Alzheimer disease defined regions. Neurol. 2018;91:e313-8. https://doi.org/10.1212/WNL.0000000000005864.

35. Pasquini L, Scherr M, Tahmasian M, Meng C, Myers NE, Ortner M, et al. Link between hippocampus' raised local and eased global intrinsic connectivity in AD. Alzheimers Dement. 2015;11:47584. https://doi.org/10.1016/j.jalz.2014.02.007.

36. Bakker A, Krauss GL, Albert MS, Speck CL, Jones LR, Stark CE, et al. Reduction of hippocampal hyperactivity improves cognition in amnestic mild cognitive impairment. Neuron. 2012;74:467-74. https://doi.org/10.1016/j.neuron.2012.03.023.

37. Scherr M, Pasquini L, Benson G, Nuttall R, Gruber M, Neitzel J, et al. Decoupling of local metabolic activity and functional connectivity links to amyloid in Alzheimer's disease. J Alzheimer's Dis : JAD. 2018;64:405-15. https://doi.org/10.3233/jad-180022.

38. Klupp E, Grimmer T, Tahmasian M, Sorg C, Yakushev I, Yousefi $\mathrm{BH}$, et al. Prefrontal hypometabolism in Alzheimer disease is related to longitudinal amyloid accumulation in remote brain regions. J Nucl Med. 2015;56:399-404. https://doi.org/10.2967/ jnumed.114.149302.

39. LaFerla FM, Oddo $S$. Alzheimer's disease: $A \beta$, tau and synaptic dysfunction. Trends Mol Med. 2005;11:170-6.

40. Spires-Jones TL, Hyman BT. The intersection of amyloid beta and tau at synapses in Alzheimer's disease. Neuron. 2014;82:756-71.

41. Schultz AP, Chhatwal JP, Hedden T, Mormino EC, Hanseeuw BJ, Sepulcre J, et al. Phases of hyperconnectivity and hypoconnectivity in the default mode and salience networks track with amyloid and tau in clinically normal individuals. J Neurosci.
2017;37:4323-31. https://doi.org/10.1523/jneurosci.3263-16. 2017.

42. Jones DT, Graff-Radford J, Lowe VJ, Wiste HJ, Gunter JL, Senjem ML, et al. Tau, amyloid, and cascading network failure across the Alzheimer's disease spectrum. Cortex; a journal devoted to the study of the nervous system and behavior. 2017;97:143-59. https://doi.org/10.1016/j.cortex.2017.09.018.

43. Cho H, Choi JY, Lee SH, Lee JH, Choi Y-C, Ryu YH, et al. Excessive tau accumulation in the parieto-occipital cortex characterizes early-onset Alzheimer's disease. Neurobiol Aging. 2017;53:10311. https://doi.org/10.1016/j.neurobiolaging.2017.01.024.

44. Low A, Mak E, Malpetti M, Chouliaras L, Nicastro N, Su L, et al. Asymmetrical atrophy of thalamic subnuclei in Alzheimer's disease and amyloid-positive mild cognitive impairment is associated with key clinical features. Alzheimer's \& Dementia: Diagnosis, Assessment \& Disease Monitoring. 2019;11:690-9. https://doi. org/10.1016/j.dadm.2019.08.001.

45. Choudhury P, Savica R, Radford JG, Duffy JR, Machulda MM, Schwarz CG, et al. Asymmetric caudate hypometabolism in the absence of frontal involvement on FDG-PET in patients with semantic dementia and behavioral symptoms. Alzheimer's \& Dementia. 2020;16:e045393. https://doi.org/10.1002/alz.045393.

46. Jacobs HIL, Van Boxtel MPJ, Jolles J, Verhey FRJ, Uylings HBM. Parietal cortex matters in Alzheimer's disease: an overview of structural, functional and metabolic findings. Neurosci Biobehav Rev. 2012;36:297-309. https://doi.org/10.1016/j.neubiorev.2011. 06.009 .

47. Ossenkoppele R, Schonhaut DR, Scholl M, Lockhart SN, Ayakta $\mathrm{N}$, Baker SL, et al. Tau PET patterns mirror clinical and neuroanatomical variability in Alzheimer's disease. Brain : a J Neurol. 2016;139:1551-67. https://doi.org/10.1093/brain/aww027.

48. Hoenig MC, Bischof GN, Seemiller J, Hammes J, Kukolja J, Onur OA, et al. Networks of tau distribution in Alzheimer's disease. Brain : a J Neurol. 2018;141:568-81. https://doi.org/10.1093/ brain/awx353.

49. Betzel RF, Byrge L, He Y, Goni J, Zuo XN, Sporns O. Changes in structural and functional connectivity among resting-state networks across the human lifespan. Neuroimage. 2014;102(Pt 2):345-57. https://doi.org/10.1016/j.neuroimage.2014.07.067.

Publisher's Note Springer Nature remains neutral with regard to jurisdictional claims in published maps and institutional affiliations. 


\section{Authors and Affiliations}

\section{Somayeh Maleki Balajoo ${ }^{1,2,3} \cdot$ Farzaneh Rahmani $^{4} \cdot$ Reza Khosrowabadi $^{5} \cdot$ Chun Meng $^{6} \cdot$ Simon B. Eickhoff2,3 . Timo Grimmer ${ }^{7}$ Mojtaba Zarei ${ }^{1,8,9} \cdot$ Alexander Drzezga ${ }^{10,11,12} \cdot$ Christian Sorg $^{7,13,14} \cdot$ Masoud Tahmasian $^{1,2,3}$ (1)}

1 Institute of Medical Science and Technology, Shahid Beheshti University, Tehran, Iran

2 Institute of Systems Neuroscience, Heinrich Heine University Düsseldorf, Düsseldorf, Germany

3 Institute of Neuroscience and Medicine, Brain \& Behaviour (INM-7), Research Centre Jülich, Jülich, Germany

4 Department of Radiology, Washington University School of Medicine, St. Louis, MO 63110, USA

5 Institute for Cognitive and Brain Sciences, Shahid Beheshti University, Tehran, Iran

6 The Clinical Hospital of Chengdu Brain Science Institute, MOE Key Laboratory for Neuroinformation, Center for Information in Medicine, School of Life Science and Technology, University of Electronic Science and Technology of China, Chengdu 611731, China

7 Department of Psychiatry and Psychotherapy, Klinikum Rechts Der Isar, Technical University of Munich, Munich, Germany
8 Department of Neurology, Odense University Hospital, Odense, Denmark

9 Department of Clinical Research, University of Southern Denmark, Odense, Denmark

10 Department of Nuclear Medicine, Faculty of Medicine and University Hospital Cologne, University of Cologne, Cologne, Germany

11 German Center for Neurodegenerative Diseases (DZNE), Bonn-Cologne, Germany

12 Institute of Neuroscience and Medicine (INM-2), Molecular Organization of the Brain, Forschungszentrum Jülich, Jülich, Germany

13 Department of Neuroradiology, Klinikum Rechts Der Isar, Technische Universität München, Munich, Germany

14 Klinikum Rechts Der Isar, TUM-Neuroimaging Center (TUM-NIC), TechnischeUniversitätMünchen, Munich, Germany 